\title{
Research on the Application of RFID in Chinese Healthcare
}

\author{
Jing Zhang* \\ International Business School \\ Yunnan University of Finance and Economics \\ Kunming, China \\ 154128175@qq.com
}

\author{
Xin Li \\ Students Affair Department \\ Yunnan University of Finance and Economics \\ Kunming, China \\ 545323361@qq.com
}

\begin{abstract}
Radio frequency identification is often referred to by the acronym RFID. Today, "RFID is used for automatic toll collection, access control, security, equipment tracking, payment at gas pumps, fast food establishments, and other retail outlets, and a wide range of other applications" [5]. RFID, as the first important technology of the twenty-first century, had a profound impact on a lot of things. Although the technology appears to be quite recent, in fact, RFID in different forms has been around for well over a century. China will attach importance to develop various advanced technologies of information platform environment for new sensors, barcode auto-identification and RFID labels, etc. This essay will look specifically in the healthcare aspect of RFID application in China. The main area of the implementation is divided into three parts, and two challenges will be addressed subsequently. The future potential for RFID in China will be demonstrated at the end of the report.
\end{abstract}

Keywords—technology; RFID; healthcare

\section{INTRODUCTION}

With more than two billion terminals in commercial operation world-wide, wireless and mobile technologies have enabled a first wave of pervasive communication systems and applications [9]. RFID (Radio frequency identification, is often referred to by the acronym RFID), as the first important technology of the twenty-first century, had a profound impact on a lot of things including television, computers, and even air travel. This essay will give the literature review first, and then analyze RFID in China, based on these; the application of RFID in Chinese healthcare will be addressed specifically, meanwhile, some differences of application between China and United States will be demonstrated. Finally, both challenge and future potential for RFID in China will be illustrated at the end of the report.

\section{LITERATURE REVIEW}

RFID is the acronym of Radio Frequency Identification. Although the technology appears to be quite recent, in fact, RFID in different forms has been around for well over a century. According to Geodan [5], "RFID is used for automatic toll collection, access control, security, equipment tracking, payment at gas pumps, fast food establishments, and other retail outlets, and a wide range of other applications". According to Simson and Beth, "the British pioneered RFID during World War II to identify their own aircraft returningfrom sorties over occupied Europe, and in the late 1960s, the US government began using RFID to tag and monitor nuclear and other hazardous materials" [10]. According to $\mathrm{Lu}$ [9], RFID has a long history, and it is part of the technological revolution both current and past. It enables quick payment of tolls and quick identification of items; furthermore, it also provides benefits, such as tracking assets, monitoring conditions for safety, and helping to prevent counterfeiting. RFID plays an integral part in the technological revolution along with the internet and mobile devices, which are connecting the world together.

\section{RFID IN CHINA}

China will attach importance to develop various advanced technologies of information platform environment for new sensors, barcode auto-identification and RFID labels, etc. "In China, RF spectrums are controlled by the National Radio Administration Bureau under the Ministry of Information Industry" (Jerry et al. 2007, p.381). Just as natural resources, radio frequencies are an important foundation for survival and development of the society in China. As China takes an active and open attitude toward planning and usage of RFID frequencies, government agencies work with standards and technical organizations to understand and support frequency planning.

\section{A. Standard}

With several Chinese government agencies having competing interest in RFID, it is difficult to understand who is in charge. "While China's standardization work is centrally managed by the Standardization Administration of China, specific standardization work is performed by affiliated relevant technical committees" [7]. The Ministry of Science and Technology took the lead in creating an association of 14 ministries and commissions to organize specialists all over China to prepare a white paper for China technical policy on RFID [7]. This white paper has been completed and submitted to the leaders of relevant ministries and commissions for examination. The white paper studies and establishes China's technological strategy for RFID, industrialization promotion strategy, and standards strategy, and it will play an important role in China's RFID development.

\footnotetext{
* Corresponding author
} 


\section{B. Main RFID implementations in China}

For improving manufacturing activities and supply chain operations, China is well aware of the potential of RFID technology. There are five main implementations of RFID have been taken place in China. The first one is China newgeneration smart ID card. It began in April 2004, China started a project to change 1 billion traditional paper ID cards for its citizens into smart card, and this could be the largest RFID card implication in history [2]. The second one is China railway automatic train identification system. Since 1999, this system has been "widely used in many railway bureaus, and more than 30,000 locomotives and 600,000 carriages have been installed with RFID tags" [2]. Another one is fixed assets management. This application solves routine inspection and maintenance problems for a great number of large-scale fixed assets in an effective manner through on-site identification using RFID. The forth one is clothes processing industries. This system enabled automatic control and the tracking of all material used in production. The last one is RFID on containers. "To enhance the management level of container transportation, a Shanghai port operator implemented RFID for container identification in 2002, at that time, this application was on the leading edge of RFID technology" [2]. Besides these five main implementations, China also pays more attention on the future development of RFID in healthcare, and the application of healthcare will become another main implementation of RFID in the coming three years [8].
The healthcare industry is a major component of the world economy, about $14 \%$ of the world's gross domestic product (GDP) is devoted to it [10]. Healthcare is an indispensable component of human welfare in the modern society. Healthcare organizations from time to time spend a tremendous amount of resources to explore and improve their services, facilities, and operational systems. "In general, an effective and efficient healthcare system is achieved through adopting new technologies, improving surgical and medical procedures, and providing comprehensive training to staff across the health spectrum" [7]. Technology plays a vital role in enhancing the overall performance of the healthcare industry. Over the years, the emergence of new technology such as electronic records, database systems, wireless technology, and computerized transaction systems has improved the efficiency and effectiveness of healthcare systems in different perspectives.

\section{The brief description of the healthcare systems}

As large and complex as a public hospital, a healthcare system can be generally categorized into three main components, accident and emergency, inpatient ward, and outpatient care. The accident and emergency department assesses and treats people with serious injuries and emergencies, and this department normally operates 24 hours a day, 365 days of the year [8]. Patients who need medical treatment are admitted to inpatient wards directly or from the accident and emergency department. Inpatient ward services and consultations use a term care approach and subspecialty expertise. Outpatient care concerns patients who have been discharged from a hospital and require further treatment [8]. More details of the healthcare systems have been provided in the graph below:

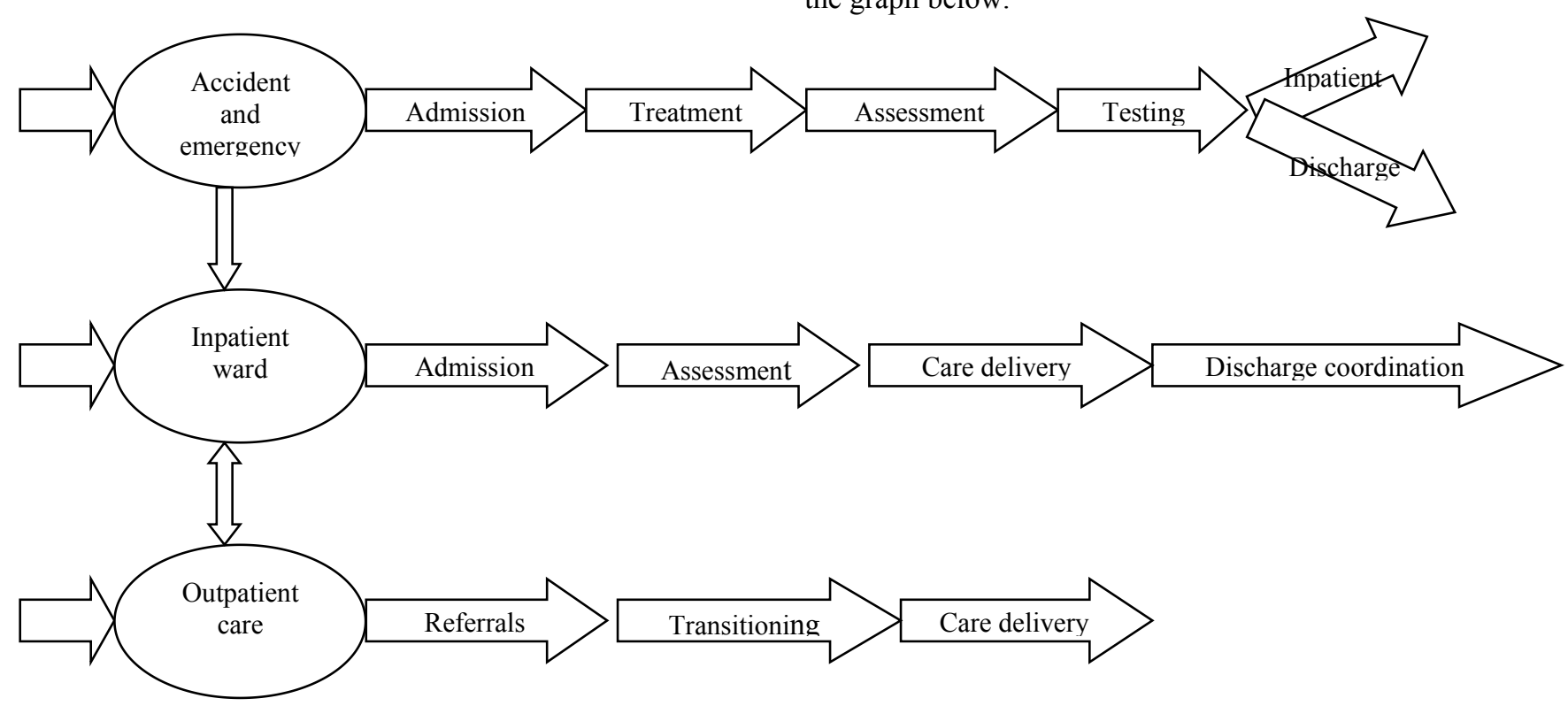

Source from: John and Leigh 2014

RFID in healthcare

The importance of healthcare

\section{Benefits of RFID in healthcare}

Healthcare is becoming one of the bright spots for the introduction of RFID applications, because of the need to minimize errors with regard to identification of patients, transplant organs, and blood. "RFID emerges as an ideal 
solution for detailed identification, and also because of the encryption capabilities of the information being transmitted between transponders and readers, HIPAA requirements are met" [13]. Today, RFID tags can be placed on sample collection bottles which can identify a lot of things, such as "the source of the sample, the time it was collected, where it has been between the patient collection point and the lab where it will be analyzed, and so on, tissue and organs can be similarly identified for cross-matching purposes to guarantee that the tagged item is used appropriately to avoid rejection" [10].

\section{FINDINGS}

Some of the applications of RFID in Chinese healthcare are being deployed today, some are being tested, and others are conceptual prototypes that are years away. The application of RFID in Chinese healthcare can be divided into three parts - tracking people and objects, safeguarding equipment and medication use, and assisting medical personnel.

\section{A. Tracking system - tracking people and objects}

In a medical emergency, minutes, or sometimes even seconds, can make the difference between life and death. An individual brought unconscious to an emergency room cannot supply healthcare providers with the critical information they may need to save his or her life, but the application of RFID'a 'smart card' containing a patient's complete identification, including medical insurance, blood type, allergies, and critical medical history, could speak for the patient in life-threatening situations" [12]. Although China is a less development country in the world, in Beijing Renai hospital, a patient management system has been founded for minority patient that is integrated with RFID technology which can collect data from various information systems and provided a real-time, on-screen, virtual floor plan of the hospital. The floor plan has icons representing individual patients. By clicking these icons, medical staff can access patients' medical history, demographic data, and current status, such as if they are scheduled for surgery or discharge. Hospital is busy environment, where people and equipment often need to be found quickly. Finding a particular piece of mobile hospital equipment, a doctor or nurse with a particular expertise or knowledge, or a particular patient is often a time-critical and urgent endeavor [6]. The main application for RFID in hospital at present in China is one closely aligned with supply chain management; here it means supply for doctors, nurses, patients, and equipments, and being able to rapidly locate them within a hospital environment at a particular time. However, only a few Chinese hospitals in major cities, such as Beijing, Shanghai, applied this technology, and used them to track people and objects. Unlike China, this technology has been broadly used in United States. For example, "the US Navy is partnering with Precision Dynamics to track wounded patients, and Doctor's Hospital in Dallas is partnering with Tenet Health systems to track newborns and ensure that they stay matched to their mothers" [2].
$B$. Equipment and medication management-safeguarding equipment and medication use

\section{1) Equipment}

In China, equipment and tools for surgical operations are normally supplied in the form of kits, which are tailored and designed for a particular operation. Since the parts have to go through a number of manufacturing and sterilization processes, it is not suitable to label them. RFID technology would be an ideal solution to this problem. "By embedding RFID tags in the parts, they can be tracked and thus can easily be reconciled before delivery to the hospital. Moreover, RFID enables medical staff to track each instrument's full history regarding involvement in patient surgical procedures" [11]. This can reduce the threat of transmitting infectious diseases and ensure that each instrument is in the right place at the right time. Nowadays, a new wave of short-range, very small RFID readers is coming online in China. This small reader has the potential to be invisible integrated into hospital equipment, particularly hospital equipment that has items dispensed or connected into it, such as blood bags, IV drips, anesthesia lines, or medication boluses. By checking the RFID tags located on the connecting item, the equipment can ensure that the correct item is being connected at the correct time for the correct patient, reducing treatment error and helping relieve the stress on nurses. However, this technology still in the testing phases in China.

\section{2) Medication}

Mismedication is a major problem in Chinese healthcare, medication errors account for $11 \%-16 \%$ of all medications, and there are at least 200,000 deaths and at least 2,500,000 injuries each year in China [6]. Medication errors were defined as the errors occurred in drug ordering, transcribing, dispensing, administering, or monitoring. The pharmaceutical industry is already moving toward a standard in which RFID tags will be placed on pill bottles in China. The current Food and Drug Administration (FDA) mandate is for all pill bottles to be tagged by 2009 [9], affording the industry an opportunity to develop solutions for medical compliance. When RFID readers are placed in the environment where medicine is kept, "the system can track whether the right medications are being taken at the right time by the right person" [9]. When a system is combined with a digital scale, as has been done at Intel Research Seattle, it can tell both which medication was taken and how much was taken [9]. This information can be provided to a nurse, a remote caregiver, or the pill taker, thus improving the quality of the user's health and reducing the strain and uncertainty on the healthcare provider, but this application still in the testing phases.

\section{Personnel management — assisting medical personnel}

RFID technology can also be useful in assisting medical personnel through the course of a procedure in China. As part of a sensor network, RFID can "infer the current state of an activity, then proactively display information, issue reminders, notify other personnel, and so forth in anticipation of the doctor's needs" [9]. At present, this is a longer-term research agenda in China. 


\section{Challenges}

Although the application of RFID in healthcare highlights a lot of advantages, it presents a host of challenges as well. There are two main challenges - radio frequency health issues and standards issues.

\section{A. Radio frequency health}

The hospital setting is a critical environment that contains a variety of radiation-emitting devices, such as x-ray machines, microwave sterilizers, and pacemakers. "Interference concerns can prevent beneficial technologies from being deployed" [10] For example, partially because of concerns over electromagnetic interference (EMI), personal cell phones are banned in most hospital setting (Simson and Beth 2006). However, this ban is not universal, and no comprehensive guidelines for EMI currently exist in China. "Although there is concern, and some evidence of EMI problems with pacemakers, electronic wheelchairs, and apnea machines, the risk seems slight with devices that have proper shielding" [10] On the other hand, the use of RFID in the medical setting must be taken on a case-by-case basis, and the benefit of the technology must be weighed against the possible risks, even though those risks might be small. It is safe to say that shortrange RFID readers pose less of a risk than long-range readers and should be used instead of long-range readers whenever possible. RFID readers should be kept away from critical healthcare equipment and should use the lowest energy level needed to perform their function.

\section{B. Standards}

The challenges to developing standards lies in the end-toend capability that RFID makes possible, because there are potentially so many different points of contact in delivering goods such as prescription drugs, it is not clear who owns the standards process. Moreover, given that the pill bottle is often manufactured in, shipped through, and consumed in a variety of countries, additional international standards bodies will need a say. It is clear that it will be some time before a final standard is resolved.

\section{The FutUre Potential FOR RFID IN CHINA}

The low-frequency RFID market is relatively mature. Applications include access control, employee attendance monitoring, automobile theft protection, animal identification, parking lot fee determination, and many others. The highfrequency RFID market is developing rapidly. Application areas include all-in-one cards, transportation, bill and note forgery prevention, healthcare and others. Thus, there is huge development potential for the high-frequency RFID market. It is anticipated that China will become one of the largest RFID application markets in the world. With China's low manufacturing costs, it is anticipated that production facilities of some leading international RFID labels and read-write equipment manufacturers will be transferred to China. In the future, RFID technical development in China will also contribute substantially to global RFID development.

\section{CONCLUSION}

As the most important technology of the twenty-first century, RFID had a profound impact on a lot of things. In fact, however, RFID in different forms has been around for well over a century. There are five main implementations in China; they are smart ID card, train identification system, assets management, clothes processing industries, and RFID on containers. Although these five applications are very important in China, the implementation of RFID in healthcare has the big potential market. It can track people and objects, safeguarding equipment and medication use, and assist medical personnel. In the future, it is expected that China will become one of the largest RFID implementation markets in the world.

\section{ACKNOWLEDGMENT}

The authors would like to thank RMIT University for providing lots of researching materials.

\section{REFERENCES}

[1] P.S. Amenta, "Histology-Medical Online Series," 4th ed. Norwalk: Appleton \& Lange, 2006.

[2] C. Angelo, P. Giuseppina, P. Andrea, "The Digital Business Ecosystem," UK: Edward Elgar Publishing Limited, 2007.

[3] Anon, "Barcode and RFID Medication Administration System [online]," Available from: http://www.gizmag.com/go/5707, 2016.

[4] K. Finkenzeller, "RFID Handbook: Fundamentals and Applications in Contact-less Smart Cards and Identification," 2nd ed. England: West Sussex, 2003

[5] A. Geodan, "Health Care Sector Starts Test with RFID," Press release, 2005.

[6] Health Data Management, "Consultant Launches New Service Line," Health Data Management, vol. 14, 2016, pp. 33-35.

[7] B. Jerry, H.David, A.P. Manuel, G.T. Les, "RFID Applied," Canada: John Wiley \& Sons, Inc., Hoboken, New Jersey, 2007.

[8] F. John, S. Leigh, "Logistics and Retail Management," 2nd ed. United States: Kogan Page Limited, 2014.

[9] Y. Lu, Z. Yan, T.Y. Laurence, S.N. Huan, "The Internet of ThingsFrom RFID to the Next-Generation Pervasive Networked Systems," United States: Taylor \& Francis Group, 2008.

[10] G. Simson, R. Beth, "RFID Applications, Security, and Privacy," United States: Pearson Education, Inc, 2006.

[11] L.J. Smart, "Considering RFID: Benefits, limitations, and best practices," C\&RL News, vol. 12, 2005, pp. 13-16.

[12] B. Sokol, "Medical Instrument Tracking Could Reduce Health Risks," RFID Journal, RFID Technology in Healthcare Application, 2014.

[13] S. Steven, "RFID—Radio Frequency Identification," United States: The McGraw-Hill Companies, Inc, 2005. 